\section{Biologischer Leitwert}

D. Meißner ${ }^{1}$ und T. Arndt ${ }^{2}$

${ }^{1}$ Dresden, Deutschland

${ }^{2}$ Bioscientia Institut für Medizinische Diagnostik GmbH, Ingelheim, Deutschland

\section{Synonym(e) BLW}

Englischer Begriff Biological reference limit

Definition Der Biologische Leitwert ist die Quantität eines Arbeitsstoffes bzw. Arbeitsstoffmetaboliten oder die dadurch ausgelöste Abweichung eines biologischen Indikators von seiner Norm beim Menschen, die als Anhalt für die zu treffenden Schutzmaßnahmen heranzuziehen ist (Zitat aus BATListe in DFG 2017).

Beschreibung BLW werden nur für solche gefährlichen Stoffe benannt, für die infolge mangelnder Datenlage keine
BAT-Werte ( $\triangleright$ Arbeitsstoff-Toleranzwert, biologischer) aufgestellt werden können, z. B. für krebserzeugende oder krebsverdächtige Stoffe. Deshalb ist auch bei Konzentrationen unterhalb des BLW das Risiko einer Gesundheitsgefährdung nicht auszuschließen.

Für den BLW wird in der Regel eine Arbeitsstoffbelastung von maximal 8 Stunden/Tag und 40 Stunden/Woche über die Lebensarbeitszeit zugrunde gelegt. In seine Festlegung fließen arbeitsmedizinische und -hygienische Erfahrungen im Umgang mit dem Arbeitsstoff ein, die nach und nach so zu vervollständigen sind, dass letztlich $u$. a. BAT-Werte abgeleitet werden können.

\section{Literatur}

DFG (2017) Ständige Senatskommission zur Prüfung gesundheitsschädlicher Arbeitsstoffe. Mitteilung 53. MAK- und BAT-Werte-Liste 2017. Wiley-VCH, Weinheim, S 260 